\title{
The morphology of the mandibular coronoid process does not indicate that Canis lupus chanco is the progenitor to dogs
}

\author{
Luc Janssens $^{1}$ (D) $\cdot$ Rebecca Miller $^{2} \cdot$ Stefan Van Dongen $^{3}$
}

Received: 19 October 2015/Revised: 12 December 2015/Accepted: 30 December 2015/Published online: 21 January 2016

(C) The Author(s) 2016. This article is published with open access at Springerlink.com

\begin{abstract}
The domestication of wolves is currently under debate. Where, when and from which wolf sub-species dogs originated are being investigated both by osteoarchaeologists and geneticists. While DNA research is rapidly becoming more active and popular, morphological methods have been the gold standard in the past. But even today morphological details are routinely employed to discern archaeological wolves from dogs. One such morphological similarity between Canis lupus chanco and dogs was published in 1977 by Olsen and Olsen. This concerns the "turned back" anatomy of the dorsal part of the vertical ramus of the mandible that was claimed to be specific to domestic dogs and Chinese wolves C. lupus chanco, and "absent from other canids". Based on this characteristic, C. lupus chanco was said to be the progenitor of Asian and American dogs, and this specific morphology has been continuously used as an argument to assign archaeological specimens, including non-Asian and non-American, to the dog clade. We challenged this statement by examining 384
\end{abstract}

Communicated by A. Schmidt-Rhaesa.

Luc Janssens

coati1@icloud.com

Rebecca Miller

rmiller@ulg.ac.be

Stefan Van Dongen

stefan.vandongen@uantwerpen.be

1 Department of Archaeology, Leiden University, Einsteinweg 2, 2333 CC Leiden, The Netherlands

2 Service of Prehistory, University of Liège, quai Roosevelt, 1, 4000 Liège, Belgium

3 Department of Evolutionary Ecology, University of Antwerp, Groenenborgerlaan 171, 2020 Antwerp, Belgium dog skulls of 72 breeds and 60 skulls of four wolf subspecies. Only $20 \%$ of dog mandibles and $80 \%$ of C. lupus chanco showed the specific anatomy. In addition, $12 \%$ of Canis lupus pallipes mandibles showed the "turned back" morphology. It can be concluded that the shape of the coronoid process of the mandible cannot be used as a morphological trait to determine whether a specimen belongs to a dog or as an argument in favour of chanco as the progenitor to dogs.

Keywords Dog - Wolf · Domestication - Morphology · Canis lupus chanco $\cdot$ Mandible $\cdot$ Coronoid process

\section{Introduction}

The domestication of wolves into dogs is an active topic of research (Boudadi-Maligne and Escarguel 2014; Germonpré et al. 2009; Larson et al. 2012; Morey and Jaeger 2015; Thalmann et al. 2013). Where, when and from which progenitor wolf sub-species dogs originated has been investigated both by osteoarchaeologists (Aaris-Sørensen 1977; Benecke 1987, 1994; Boudadi-Maligne and Escarguel 2014; Huxley 1880; Iljin 1941; Nehring 1888; Rütimeyer 1861; Stockhaus 1965; Studer 1901; Sumiński 1975) and geneticists (Anderson et al. 2009; Ardalan et al. 2011; Axelsson et al. 2013; Brown et al. 2011; Freedman et al. 2014; Gundry et al. 2007; Ho et al. 2005; Irion et al. 2003; Karlsson et al. 2007; Khosravi et al. 2013; Kirkness et al. 2003; Klütsch and de Caprona 2010; Larson and Burger 2013; Leonard et al. 2002; Lindblad-Toh et al. 2005; Ostrander and Wayne 2005; Pang et al. 2009; Savolainen et al. 2002, 2004; Schmutz and Berryere 2007; Schoenebeck and Ostrander 2013; Thalmann et al. 2013; Tsuda et al. 1997; Vaysse et al. 2011; Verginelli et al. 
2005; Vila et al. 1999, 2005; Vilà et al. 1993, 1997; Vonholdt et al. 2010; Wayne 2012; Wayne and Ostrander 1999, 2007).

Briefly there are two current views. One group of researchers proposes an origin of dogs after the Last Glacial Maximum (LGM) in Europe and during the Magdalenian, about 18,000 years ago (Thalmann et al. 2013). This evidence is based on genetic research (Ho et al. 2005; Thalmann et al. 2013), and the morphology of canine archaeological remains that is distinctively smaller than those of wolves (Altuna et al. 1984; Boudadi-Maligne and Escarguel 2014; Boudadi-Maligne et al. 2012; Célérier 1994; Célérier et al. 1999; Chaix 2000; Larson and Burger 2013; Leesch et al. 2012; Morel and Müller 1997; Napierala and Uerpmann 2012; Pionnier-Capitan 2010; Pionnier-Capitan et al. 2011; Street 2002).

The other group claims that dogs originated before the LGM, as early as in the Aurignacian and Gravettian and thus 35,000 years ago (Bocherens et al. 2014; Germonpré et al. 2009, 2012; Ovodov et al. 2011; Sablin and Khlopachev 2002). Although genetic analysis has not found any relationship between these old archaeological canine specimens (Thalmann et al. 2013) purported to be domesticated wolves and modern dogs, these researchers suggest that these animals were, however, domesticated, but did not produce surviving offspring (aborted domestication waves) (Germonpré et al. 2012; Skoglund et al. 2011). The arguments to place these pre-LGM specimens in the dog clade are based on morphology alone and mainly on wider and shorter snouts. Drake et al. (2015) have, however, demonstrated that this criterion (shorter and wider snouts) is not useful in distinguishing dogs from wolves and also identified some of the so-called pre-LGM dog fossils as wolves.

Many morphological differences have been described between wolves and dogs in the literature since the eighteenth century (Clutton-Brock 1962; Degerbøl 1961; Nehring 1888; Stockhaus 1965; Studer 1901; Wolfgram 1894). Three morphological methods were used to examine morphological differences:

- The "obvious" visual difference in appearance (morphology, sensu stricto) (Olsen and Olsen 1977).

- The difference in size (morphometry) (Benecke 1987, 1994; Boudadi-Maligne and Escarguel 2014; Napierala and Uerpmann 2012).

- The difference in appearance (form) that cannot be recognized visually with certainty (geometric morphometrics) (e.g., Drake and Klingenberg 2010; Milenkovic et al. 2010; Pionnier-Capitan 2010; Schmitt and Wallace 2012).
The most frequently reported morphological and morphometric differences used to distinguish dogs from wolves are smaller stature and thus smaller anatomical parts (e.g., skull, teeth such as carnassials, etc.), shorter and wider snouts, tooth crowding, larger orbital angles and a "turned back" morphology of the dorsal side (apex) of the vertical ramus of the mandible (coronoid process) (Olsen and Olsen 1977, Fig. 1 and 2, p. 534-535). The latter morphological difference is based purely on difference in shape. This distinctive morphological characteristic was described in 1977 by Olsen and Olsen (Olsen and Olsen 1977). The authors state that the origin of Asian and American (New World) dogs must have originated in the Far East and proposed the Tibetan wolf (Chinese wolf, Asian wolf, Canis lupus chanco) as the dog's ancestor (Olsen and Olsen 1977, 534). This opinion was based on the specific "turned back" morphology of the coronoid process of the mandible, claimed to be "specific to domestic dogs" and Chinese wolves, and to be "absent from other canids" (Olsen and Olsen 1977, 534). Based on this assumption, $C$. lupus chanco was said to be "the progenitor of dogs", and this specific morphological trait is still used in recent publications to assign archaeological specimens to the dog clade (e.g., Ovodov et al. 2011).

We tested the statement of Olsen and Olsen (1977) by examining 384 dog mandibles of many breeds, of which six breeds are Asian or American, and 60 wolf mandibles of four sub-species. Our aim is to examine whether this "turned back" morphology is indeed present in "all" dogs and only in C. lupus chanco as hypothesized.

\section{Materials and methods}

All examined mandibles are from reputable museum collections. These had been collected in historical and recent periods and were professionally prepared. All are intact and from adult animals. In total $444 \mathrm{dog}$ and wolf skulls were examined (888 mandibles) including $384 \mathrm{dog}$ skulls and 60 wolf skulls. For the wolves (Table 1), 37 are from the collection of The George S. Wise Faculty of Life Sciences, Department of Zoology at Tel-Aviv University, Israel (ZMTAU). Thirty-two of these were Canis lupus pallipes and five Canis lupus arabs. Seven skulls were examined from the collection of the Natural History Museum in London, Great Britain (BMNH): six C. lupus arabs, and one $C$. lupus pallipes. Eleven skulls are from the collection of the Natural History Museum Bern, Switzerland (NMBE), all from Eurasian wolves (Canis lupus lupus) from Central Europe or Russia. Five specimens of $C$. lupus chanco from the collection of the Department of Vertebrate Zoology, Smithsonian Institution at the National Museum 
Table 1 List of wolf skulls used in this study

\begin{tabular}{|c|c|c|c|c|}
\hline Museum ID & Genus & Species & $\begin{array}{l}\text { Sub- } \\
\text { species }\end{array}$ & Region \\
\hline BMNH ZD.1891.2.5.1 & Canis & lupus & arabs & Bouraida \\
\hline BMNH ZD.1895.10.8.1 & Canis & lupus & arabs & Aden \\
\hline BMNH ZD.1899.11.6.36 & Canis & lupus & arabs & Muscat \\
\hline BMNH ZD.1924.8.13.1 & Canis & lupus & arabs & Jeddah \\
\hline BMNH ZD.1940.193 & Canis & lupus & pallipes & ? \\
\hline BMNH ZD.1948.368 & Canis & lupus & pallipes & $?$ \\
\hline BMNH ZD.1897.1.14.4 & Canis & lupus & arabs & Jaquakar \\
\hline NMBE1028185 & Canis & lupus & lupus & Russia \\
\hline NMBE1028188 & Canis & lupus & lupus & Russia \\
\hline NMBE1028189 & Canis & lupus & lupus & Russia \\
\hline NMBE1028192 & Canis & lupus & lupus & Poland \\
\hline NMBE1028193 & Canis & lupus & lupus & Russia \\
\hline NMBE1028204 & Canis & lupus & lupus & Poland \\
\hline NMBE1028205 & Canis & lupus & lupus & Poland \\
\hline NMBE1028206 & Canis & lupus & lupus & Poland \\
\hline NMBE1028207 & Canis & lupus & lupus & Poland \\
\hline NMBE1028209 & Canis & lupus & lupus & Poland \\
\hline NMBE1028211 & Canis & lupus & lupus & Russia \\
\hline USNM00607 & Canis & lupus & chanco & China \\
\hline USNM00610 & Canis & lupus & chanco & China \\
\hline USNM00613 & Canis & lupus & chanco & China \\
\hline USNM00616 & Canis & lupus & chanco & China \\
\hline USNM00619 & Canis & lupus & chanco & China \\
\hline ZMTAU 09439 & Canis & lupus & pallipes & Golan \\
\hline ZMTAU 09460 & Canis & lupus & arabs & Sandiya \\
\hline ZMTAU 10334 & Canis & lupus & pallipes & Galilei \\
\hline ZMTAU 10338 & Canis & lupus & pallipes & Galilei \\
\hline ZMTAU 10355 & Canis & lupus & pallipes & Golan \\
\hline ZMTAU 10402 & Canis & lupus & pallipes & Golan \\
\hline ZMTAU 10608 & Canis & lupus & pallipes & Galilei \\
\hline ZMTAU 10609 & Canis & lupus & pallipes & Golan \\
\hline ZMTAU 10610 & Canis & lupus & pallipes & Golan \\
\hline ZMTAU 10615 & Canis & lupus & pallipes & Golan \\
\hline ZMTAU 10619 & Canis & lupus & pallipes & Golan \\
\hline ZMTAU 10621 & Canis & lupus & pallipes & Golan \\
\hline ZMTAU 10682 & Canis & lupus & pallipes & Golan \\
\hline ZMTAU 10685 & Canis & lupus & pallipes & Golan \\
\hline ZMTAU 10686 & Canis & lupus & pallipes & Golan \\
\hline ZMTAU 10688 & Canis & lupus & pallipes & Golan \\
\hline ZMTAU 10692 & Canis & lupus & pallipes & Golan \\
\hline ZMTAU 11041 & Canis & lupus & pallipes & Galilei \\
\hline ZMTAU 11109 & Canis & lupus & pallipes & Galilei \\
\hline ZMTAU 11110 & Canis & lupus & pallipes & Golan \\
\hline ZMTAU 11118 & Canis & lupus & pallipes & Galilei \\
\hline ZMTAU 11119 & Canis & lupus & pallipes & Golan \\
\hline ZMTAU 11121 & Canis & lupus & pallipes & Golan \\
\hline ZMTAU 11250 & Canis & lupus & pallipes & Galilei \\
\hline
\end{tabular}

Table 1 continued

\begin{tabular}{lllll}
\hline Museum ID & Genus & Species & $\begin{array}{l}\text { Sub- } \\
\text { species }\end{array}$ & Region \\
\hline ZMTAU 11275 & Canis & lupus & pallipes & Galilei \\
ZMTAU 11417 & Canis & lupus & pallipes & Galilei \\
ZMTAU 11418 & Canis & lupus & pallipes & Golan \\
ZMTAU 11475 & Canis & lupus & arabs & Negev \\
ZMTAU 11476 & Canis & lupus & pallipes & Golan \\
ZMTAU 11479 & Canis & lupus & pallipes & Galilei \\
ZMTAU 11516 & Canis & lupus & pallipes & Golan \\
ZMTAU 11685 & Canis & lupus & pallipes & Golan \\
ZMTAU 12130 & Canis & lupus & pallipes & Galilei \\
ZMTAU 12130-2 & Canis & lupus & arabs & Negev \\
ZMTAU 12251 & Canis & lupus & arabs & Negev \\
ZMTAU 12254 & Canis & lupus & arabs & Muscat \\
ZMTAU 12279 & Canis & lupus & arabs & Negev \\
\hline
\end{tabular}

Sub-species, institute and accession numbers (ID) are reported. BMNH: British Museum of Natural History. NMBE: Natural History Museum Bern, Switzerland, USNM: Department of Vertebrate Zoology, Smithsonian Institution at the National Museum of Natural History, Washington DC, USA, ZMTAU: Department of Zoology at Tel-Aviv University, Israel

of Natural History, Washington DC, USA (USNM), were also examined.

We also examined 123 dog skulls from the collection of the anatomy department of the school for Veterinary Medicine, Ghent University, Belgium, and 261 skulls from the collection of The Museum of Natural History, Bern, Switzerland (total 384) (Table 2). The skulls belong to 72 different breeds, of which six breeds and 33 skulls are Asian or American. These are Alaskan malamute (5), Canadian Eskimo dog (4), Chow-Chow (16), Shar Pei (1), Tibetan Mastiff (6) and Tibetan Terrier (1).

Each mandible was digitally photographed from a distance of 40-50 cm with a digital Nikon D 700 camera with a $50 \mathrm{~mm}$ lens. The photographs were imported in the OsiriX Imaging Software program. A straight vertical line was then drawn confluent with the straight part of the ventral caudal border of the mandible. The mandibles were divided in two categories based on the morphology of the coronoid process and by drawing a straight line (green on the figures) coinciding with the caudal border. For Category 1 , the mandible has a perfect vertical straight caudal border (Fig. 1) or the uppermost part of the apex points minimally in the caudal direction, while the caudal border is straight (Fig. 2). In this category, the straight green line follows the caudal bony border of the vertical ramus and the dorsal aspect of the mandible does not cross the green line or transects only a very small part at the tip. For Category 2, the caudal border is concave over its entire length and has the form of a dolphin fin (Fig. 3). Here, the 
Table 2 Dog skulls used in this study grouped alphabetically by breed

\begin{tabular}{|c|c|c|c|c|c|}
\hline Breed & $\mathrm{Nr}$ & TB & Breed & $\mathrm{Nr}$ & TB \\
\hline Afghan hound & 13 & 2 & Greyhound & 10 & 1 \\
\hline Airedale terrier & 4 & 1 & $\begin{array}{l}\text { Groenendael Belgian } \\
\text { shepherd }\end{array}$ & 18 & 1 \\
\hline Akita Inu & 8 & 1 & Hahoawu & 1 & \\
\hline $\begin{array}{l}\text { Alaskan } \\
\text { Malamute }\end{array}$ & 5 & 2 & Irish setter & 2 & \\
\hline Barzoi & 11 & 2 & Irish wolfhound & 8 & 2 \\
\hline Basenji & 1 & & Jagdterrier & 2 & \\
\hline Batak hound & 11 & 3 & Karelian Bear dog & 18 & 3 \\
\hline Beagle & 9 & 2 & Kuvasc & 1 & \\
\hline Bearded collie & 1 & & Labrador retriever & 13 & 2 \\
\hline Berger de Brie & 1 & & Leonberger & 1 & \\
\hline $\begin{array}{l}\text { Berner } \\
\text { Sennenhund }\end{array}$ & 32 & 4 & Lundehund & 2 & \\
\hline Bloodhound & 7 & 1 & $\begin{array}{l}\text { Malinois Belgian } \\
\text { shepherd }\end{array}$ & 2 & 1 \\
\hline Border collie & 5 & 3 & Mastino Napolitano & 1 & \\
\hline $\begin{array}{l}\text { Bouvier des } \\
\text { Flandres }\end{array}$ & 4 & 2 & Mayar Agar & 2 & 1 \\
\hline Boxer & 2 & & Pariah hound & 10 & 2 \\
\hline Bull terrier & 1 & & Pembroke Welsh Corgi & 1 & \\
\hline Canaan dog & 1 & & Pharaoh hound & 4 & \\
\hline $\begin{array}{l}\text { Canadian Eskimo } \\
\text { dog }\end{array}$ & 4 & & Pointer & 1 & 1 \\
\hline Chow Chow & 16 & 3 & Poodle & 6 & 2 \\
\hline Cocker spaniel & 4 & & Rhodesian Ridgeback & 2 & 2 \\
\hline Crossbred & 5 & 3 & Rottweiler & 3 & \\
\hline Dalmatian & 1 & & Saint Bernhard & 2 & \\
\hline Dingo & 3 & 2 & Saluki & 2 & \\
\hline $\begin{array}{l}\text { Doberman } \\
\text { pinscher }\end{array}$ & 15 & 5 & Samojeed & 8 & 2 \\
\hline Entelbucher & 1 & & Scottish collie & 1 & \\
\hline Finnish spitz & 3 & 1 & Scottish terrier & 16 & \\
\hline Flatcoat retriever & 1 & & Shar Pei & 1 & \\
\hline Fox terrier & 1 & & Siberian Husky & 14 & 3 \\
\hline Gaint schnauzer & 1 & & Sloughi & 1 & \\
\hline Galgo Espanjol & 2 & & Swiss shepherd & 1 & \\
\hline German braque & 3 & 1 & $\begin{array}{l}\text { Tervueren Belgian } \\
\text { shepherd }\end{array}$ & 5 & \\
\hline German shepherd & 10 & 3 & Tibetan Mastiff & 6 & 1 \\
\hline Golden retriever & 6 & 1 & Tibetan spaniel & 1 & \\
\hline Great Dane & 2 & & Weimaraner & 1 & \\
\hline Great spitz & 7 & 2 & Whippet & 4 & 2 \\
\hline Greenland dog & 10 & 1 & Wolfspitz & 2 & 1 \\
\hline Total breeds & 72 & & Total skulls & 384 & \\
\hline
\end{tabular}

In bold are New World and Asian breeds. Nr refers to the number of skulls examined. TB refers to "Turned Back" morphology

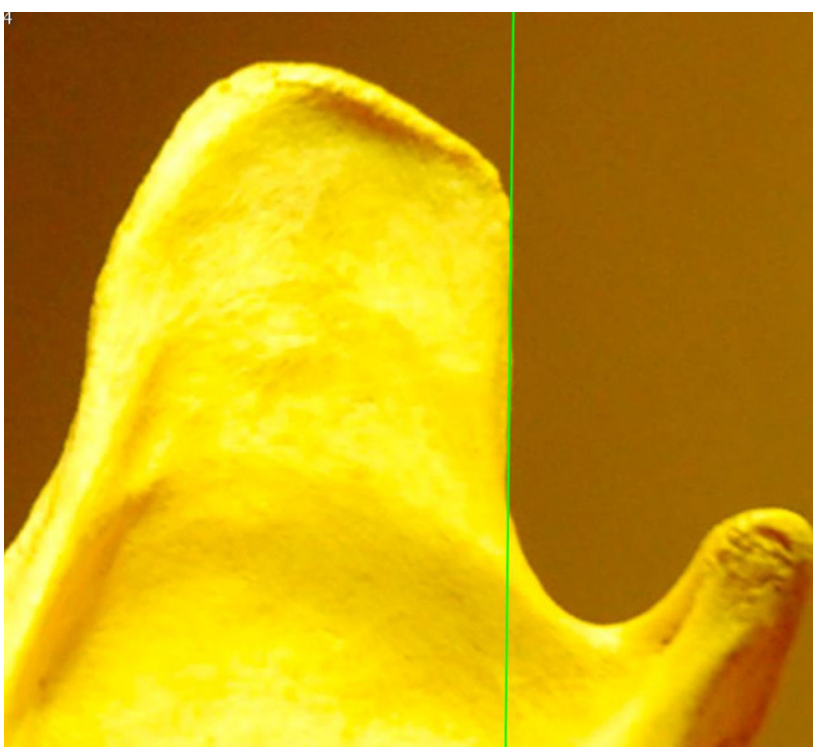

Fig. 1 Category 1: Straight caudal border of the vertical ramus. The vertical line that coincides with the ventral part of the caudal border of the vertical ramus of the mandible does not cut through the dorsal caudal ramus

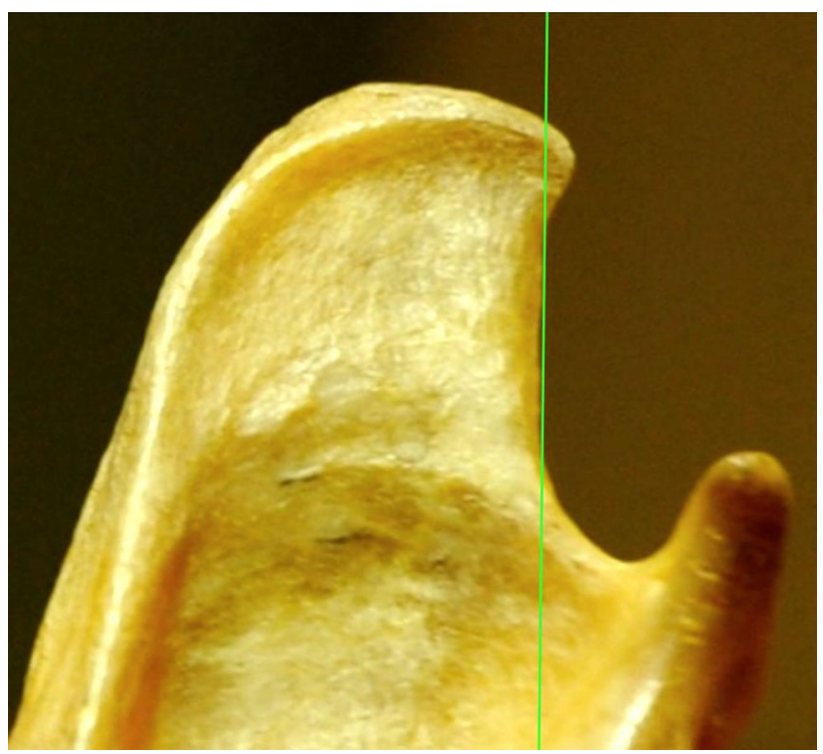

Fig. 2 Category 2: Straight caudal border with minimal tip curvature. The vertical line that coincides with the ventral part of the caudal border of the vertical ramus of the mandible coincides with the caudal border and does only cut through the tip of dorsal caudal ramus

vertical line transects most of the caudal vertical ramus and the line cannot coincide with the caudal border which is concave. 


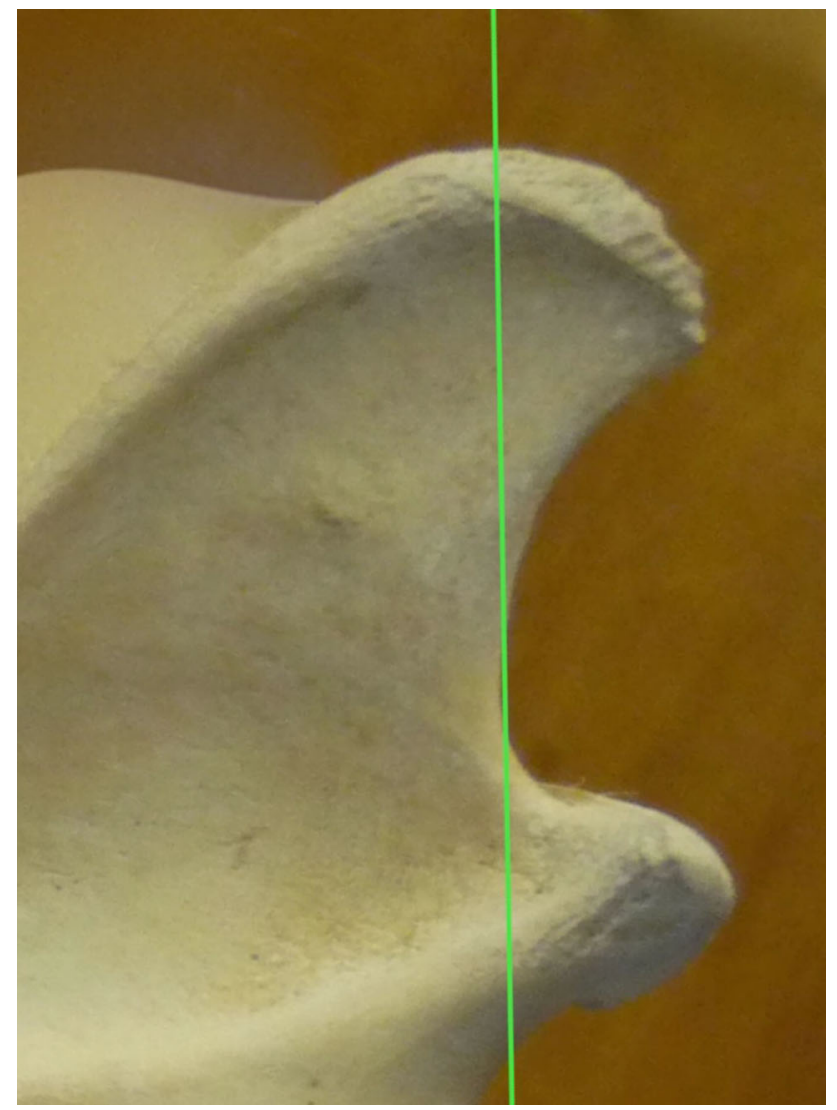

Fig. 3 Turned back morphology. The vertical line at the caudal border of the vertical ramus of the mandible does not coincide with the border and cuts through a large part of the dorsal ramus

\section{Results}

All left and right mandibles from the same skull show identical anatomy; therefore, frequencies are per skull, not mandible. Fifty-two wolf skulls had a straight caudal border $(87 \%)$, while eight (13\%) had a "turned back" morphology (Table 3). Eurasian wolves and C. lupus arabs all had straight mandibles. C. lupus pallipes had four specimens with mandibles with the "turned back" morphology (12\%) (Fig. 4) and C. lupus chanco four out of five mandibles with "turned back" morphology $(80 \%)$ but one with straight morphology (20\%) (Fig. 5).

Of the 384 dog skulls, 312 had a straight caudal border $(81 \%)$ and 72 mandibles had "turned back" morphology

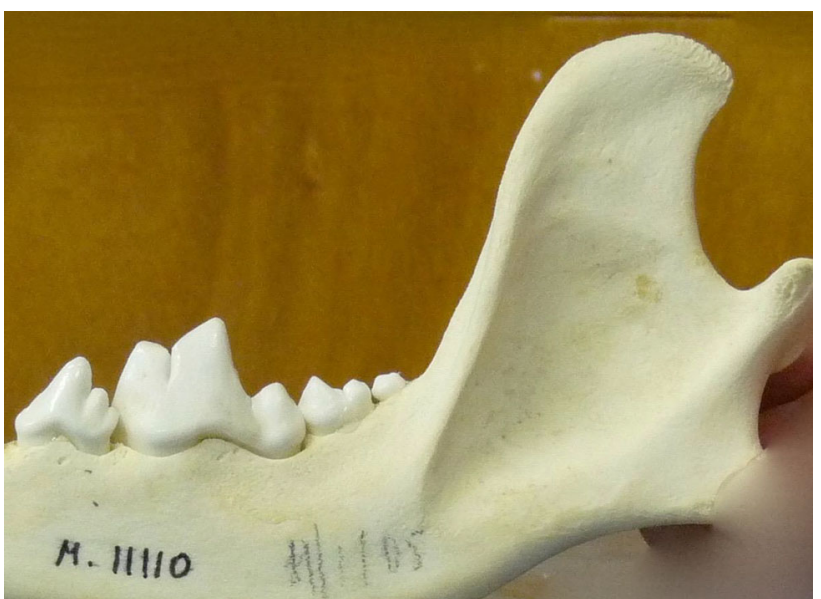

Fig. 4 A Canis lupus pallipes mandibular specimen with "turned back" morphology. Accession number ZMTAU1110 (George Wise faculty of Life Sciences, Israel)

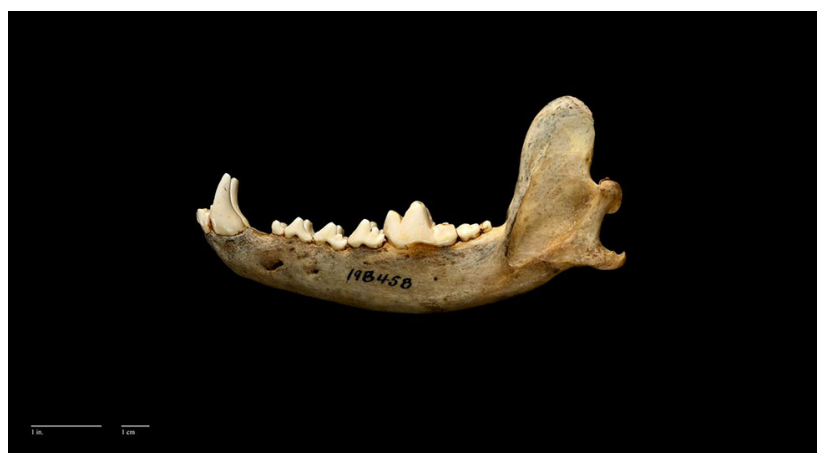

Fig. 5 The Canis lupus chanco mandibular specimen without the "turned back" morphology. Accession number 18B458- NHB 2015USNM00610 (Smithsonian Institution, USA). Photo: D. E. Hurlbert

(19\%). There was no relation between the "turned back" anatomy and breed; this was spread across 37 breeds (Table 3).

Three of seven Asian and American breeds (41 mandibles) had seven "tuned back" mandibles (17\%) so most mandibles in these breeds were straight (Fig. 6).

\section{Discussion}

Three main claims are made in Olsen and Olsen's article (1977). The first is that the Chinese wolf is progenitor to Asian and New World dogs. When Olsen and Olsen's

Table 3 Morphological categories of the coronoid process of the mandible

\begin{tabular}{|c|c|c|c|c|c|c|}
\hline & Dogs & $\begin{array}{l}\text { Canis lupus } \\
\text { pallipes }\end{array}$ & $\begin{array}{l}\text { Canis lupus } \\
\text { arabs }\end{array}$ & $\begin{array}{l}\text { Canis lupus } \\
\text { chanco }\end{array}$ & $\begin{array}{l}\text { Canis lupus } \\
\text { Eurasian }\end{array}$ & $\begin{array}{l}\text { Wolves } \\
\text { Total }\end{array}$ \\
\hline Total number & 384 & 37 & 7 & 5 & 11 & 60 \\
\hline Category 1: straight morphology & $81 \%(312)$ & $88 \%(33)$ & $100 \%(7)$ & $20 \%(1)$ & $100 \%(11)$ & 52 \\
\hline Category 2: "Turned back" morphology & $19 \%(72)$ & $12 \%(4)$ & & $80 \%(4)$ & & 8 \\
\hline
\end{tabular}



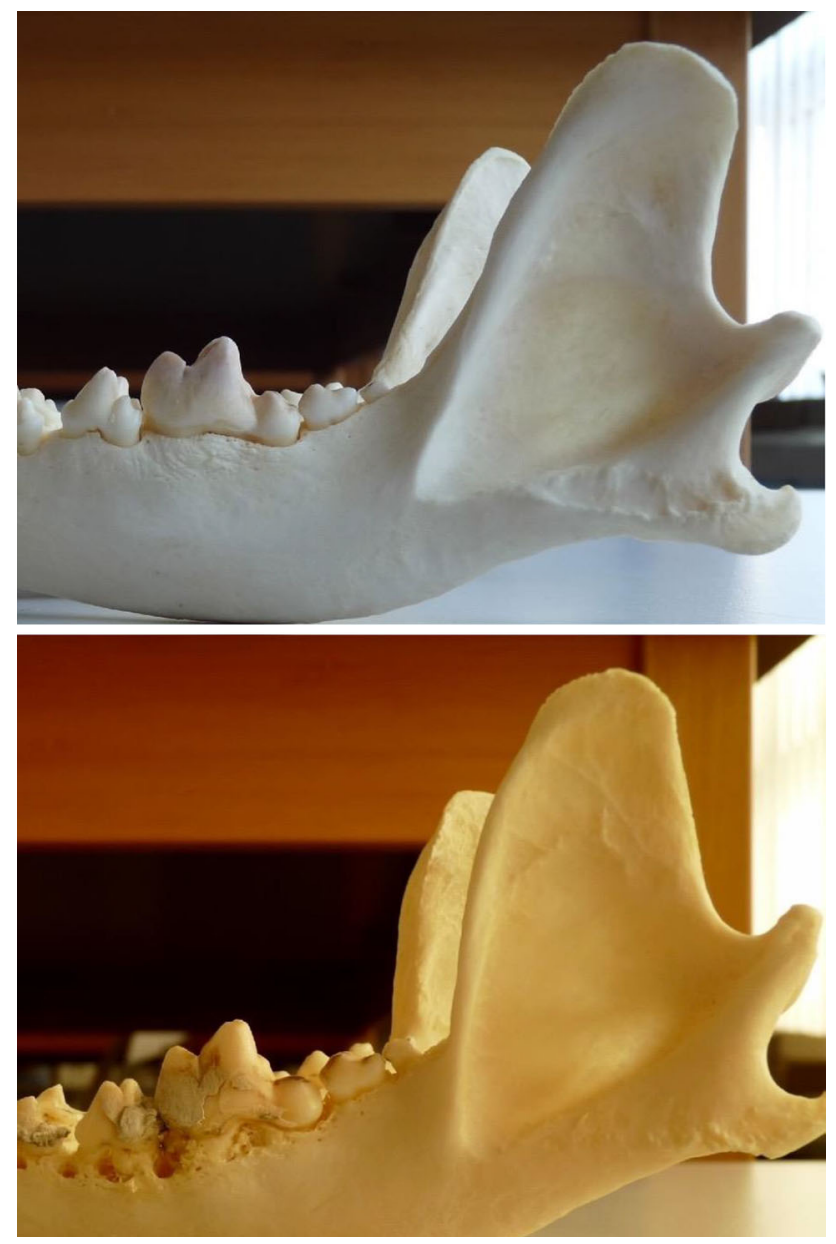

Fig. 6 A mandibular specimen of an Asian/American dog without the "turned back" morphology. Top Alaskan Malamute specimen. Accession number 1051378-313/78 (Museum of Natural History, Bern, Switzerland). Bottom Akita Inu specimen. Accession number 1051382-523/82 (Museum of Natural History, Bern, Switzerland)

article (1977) was published, it was still uncertain if only the wolf was a progenitor to dogs. In addition to the wolf, Canis aureus was said to be a possible forefather of small breed dogs (Darwin 1868; Lorenz 2002). It was also uncertain if there had been only one domestication wave, or if regional and different domestication phenomena had occurred and so for example local Asian wolves could then have been directly ancestral to Asian and New World dogs and Eurasian wolves to European dogs. The article should thus be viewed in this historical perspective. The fact that C. lupus chanco is called "the Chinese wolf" in the article, not Tibetan wolf (Pocock 1946), should also be placed in the same historical perspective as the 1970s were a period of a Sino-American rapprochement (Oksenberg 1982). Recent genetic analysis has confirmed that only wolves are progenitors to dogs, contradicting older theories about different geographic domestication waves (Duleba et al. 2015; Horard-Herbin et al. 2014; Larson et al. 2012;
Thalmann et al. 2013) and has revealed that New World dogs did not originate locally but invaded the continent together with early migration waves of Homo sapiens (Leonard et al. 2002; Savolainen et al. 2002).

The original article shows drawings of 13 mandibles of which only ten have sufficient intact anatomy to make interpretation possible (according to personal re-examination of the published drawings by LJ). Of these, six belong to dogs, one to $C$. lupus chanco and three to species other than Canis lupus. All dogs and all C. lupus chanco specimens show the "turned back" anatomy. It is not reported if more than these seven mandibles were examined. If not, it is difficult to understand why such a general statement was published. C. lupus chanco skulls are very difficult to find in zoological and natural history collections. This may explain why only one was reported in the article. We found only eleven skulls in many worldwide collections. Of these only five had intact mandibular anatomy, of which one $(20 \%)$ had a straight caudal mandibular ramus, contradicting Olsen and Olsen's (1977) original statement.

The second assertion is that the "tuned back" morphology is absent from other canids. This statement is unsupportable as we have demonstrated the presence of the "turned back" morphology in C. lupus pallipes mandibles. Studer (1901) early on reported that from all examined wolf skulls pallipes and chanco were the most anatomically similar. This may explain why these two wolf subspecies share this "turned back" morphology, unseen in the two other wolf sub-species we examined.

The third statement is that "dogs have the turned back morphology". At one point in the article this statement is made in general: "all dogs" have the turned back morphology (Olsen and Olsen 1977, 534, last paragraph), while in another location it refers to "New World and Asian dogs" (Olsen and Olsen 1977, 533, fifth paragraph), while the title of the article refers only to New World dogs. The "turned back" morphology is present in the six dog mandible drawings in the article, but the same pattern was not observed in the large group of dog mandibles we examined, not in general and not in Asian or New World dogs. Indeed, only a minority of dogs $(20 \%)$ have "turned back" morphology. In addition there are no differences in occurrence between Asian and/or New World dogs nor in the total group of dogs (18\% in these breeds vs. $20 \%$ in total).

\section{Conclusion}

The statement that all dogs have a specific "turned back" morphology of the mandibular coronoid process, and that they share this specific morphology with only one wolf sub-species (C. lupus chanco), is untenable. This morphological trait cannot therefore be used as an argument to 
claim that archaeological remains belong to dogs, nor to argue that $C$. lupus chanco is the progenitor of dogs.

Acknowledgments We would like to thank Shai Meiri and Daniel Berkowic from Israel, Roberto Portela Miguez from the Natural History Museum in London, Paul Schmid and Marc Nussbaumer from Switzerland and Paul Simoens from Belgium for allowing us to use their collections for research. We thank Kristen Quarles from the Smithsonian institution for assistance with their collection and thank Thijs Van Kolfschoten for critical comments. David Jaggar is thanked for linguistic help. We are grateful to the anonymous reviewers of the article for improvements. Finally Christien De Paepe is thanked for assistance with the graphics.

Open Access This article is distributed under the terms of the Creative Commons Attribution 4.0 International License (http://crea tivecommons.org/licenses/by/4.0/), which permits unrestricted use, distribution, and reproduction in any medium, provided you give appropriate credit to the original author(s) and the source, provide a link to the Creative Commons license, and indicate if changes were made.

\section{References}

Aaris-Sørensen K (1977) The subfossil wolf, Canis lupus L. in Denmark. Vidensk Meddr dabsk naturhistorisch Forensen 29:129-146

Altuna J, Baldeon A, Mariezkurrena K (1984) Dépôts rituels magdaléniens de la grotte d'Erralla (Pays Basque). Munibe 36:3-10

Anderson T, Candille S, Musiani M, Greco C, Stahler D, Smith D, Padhukasahasram B, Randi E, Leonard J, Bustamante C (2009) Molecular and evolutionary history of melanism in North American gray wolves. Science 323:1339-1343

Ardalan A, Kluetsch C, Zhang A, Erdogan M, Uhlén M, Houshmand M, Tepeli C, Ashtiani S, Savolainen P (2011) Comprehensive study of mtDNA among Southwest Asian dogs contradicts independent domestication of wolf, but implies dog-wolf hybridization. Ecol Evol 1:373-385

Axelsson E, Ratnakumar A, Arendt M, Maqbool K, Webster M, Perloski M, Liberg O, Arnemo J, Hedhammar A, Lindblad-Toh $\mathrm{K}$ (2013) The genomic signature of dog domestication reveals adaptation to a starch-rich diet. Nature 495:360-364

Benecke N (1987) Studies on early dog remains from Northern Europe. J Archaeol Sci 14:31-49

Benecke N (1994) Archäozoologische Studien zur Entwicklung der Haustierhaltung vol 64. Akademie Verlag Schriften zur Ur- und Frühgeschichte, Berlin

Bocherens H, Drucker D, Germonpré M, Lázničková-Galetová M, Nait Y, Wissin C, Brůžek J, Oliva M (2014) Reconstruction of the Gravettian food-web at Předmostí I using multi-isotopic tracking of bone collagen. Quat Int 249:1-18

Boudadi-Maligne M, Escarguel G (2014) A biometric re-evaluation of recent claims for early upper palaeolithic wolf domestication in Eurasia. J Archaeol Sci 45:80-89

Boudadi-Maligne M, Mallye J-B, Langlais M, Barshay-Szmidt C (2012) Magdalenian dog remains from Le Morin rock-shelter (Gironde, France). Socio-economic implications of a zootechnical innovation. PALEO Revue d'archéologie préhistorique 23:39-54

Brown SK, Pedersen P, Niels C, Jafarishorije S, Bannasch D, Ahrens K, Wu J, Okon M, Sacks B (2011) Phylogenetic distinctiveness of middle eastern and southeast Asian village dog Y chromosomes illuminates dog origins. PLoS One 6:e28496
Célérier G (1994) L'abri sous roche de Pont d'Ambon à Bourdeilles (Dordogne). Gallia préhistoire 36:65

Célérier G, Tisnerat N, Valladas H (1999) Données nouvelles sur l'âge des vestiges de chien à Pont d'Ambon, Bourdeilles (Dordogne). Paleobiology 11:163-165

Chaix L (2000) A preboreal dog from the northern Alps (Savoie, France). BAR Int Ser 889:49-60

Clutton-Brock J (1962) Near eastern canids and the affinities of the Natufian dogs. Zeitschrift für Tierzüchtung und Züchtungsbiologie 76:326-333

Darwin C (1868) The variation of animals and plants under domestication, vol 2. John Murray, London

Degerbøl M (1961) On a find of a preboreal domestic dog Canis familiaris L. from Star Carr, Yorkshire, with remarks on other Mesolithic dogs. Proc Prehist Soc (New Ser) 27:35-55

Drake AG, Klingenberg CP (2010) Large-scale diversification of skull shape in domestic dogs: disparity and modularity. Am Nat 175:289-301. doi:10.1086/650372

Drake AG, Coquerelle M, Colombeau G (2015) 3D morphometric analysis of fossil canid skulls contradicts the suggested domestication of digs during the late palaeolithic. Sci Rep. doi:10. 1038/srep08299

Duleba A, Skonieczna K, Bogdanowicz W, Malyarchuk B, Grzybowski T (2015) Complete mitochondrial genome database and standardized classification system for Canis lupus familiaris. Forensic Sci Int: Genet 19:123-129

Freedman AH, Gronau I, Schweizer R, Ortega-Del Vecchyo D, Han E, Sil P, Galaverni M, Ma P, Lorente-Galdos B (2014) Genome sequencing highlights the dynamic early history of dogs. PLoS Genet 10:e1004016

Germonpré M, Sablin MV, Stevens RE, Hedges RE, Hofreiter M, Stiller M, Després VR (2009) Fossil dogs and wolves from palaeolithic sites in Belgium, the Ukraine and Russia: osteometry, ancient DNA and stable isotopes. J Archaeol Sci 36:473-490

Germonpré M, Lázničková-Galetová M, Sablin MV (2012) Palaeolithic dog skulls at the Gravettian Předmostí site, the Czech Republic. J Archaeol Sci 39:184-202

Gundry RL, Allard MW, Moretti TR, Honeycutt RL, Wilson MR, Monson KL, Foran DR (2007) Mitochondrial DNA analysis of the domestic dog: control region variation within and among breeds. J Forensic Sci 52:562-572

Ho SY, Phillips MJ, Cooper A, Drummond AJ (2005) Time dependency of molecular rate estimates and systematic overestimation of recent divergence times. Mol Biol Evol 22:1561-1568

Horard-Herbin M-P, Tresset A, Vigne J-D (2014) Domestication and uses of the dog in western Europe from the paleolithic to the iron age. Anim Front 4:23-31

Huxley TH (1880) On the cranial and dental characters of the Canidæ. In: Proceedings of the zoological society of London. Wiley Online Library, 238-288

Iljin NA (1941) Wolf-dog genetics. J Genet 42:359-414

Irion D, Schaffer A, Famula T, Eggleston M, Hughes S, Pedersen N (2003) Analysis of genetic variation in $28 \mathrm{dog}$ breed populations with 100 microsatellite markers. J Hered 94:81-87

Karlsson EK et al (2007) Efficient mapping of mendelian traits in dogs through genome-wide association. Nat Genet 39:1321-1328

Khosravi R, Rezaei HR, Kaboli M (2013) Detecting hybridization between Iranian wild wolf (Canis lupus pallipes) and freeranging domestic dog (Canis familiaris) by analysis of microsatellite markers. Zoolog Sci 30:27-34

Kirkness EF, Baranowska I, Wade C, Hillbertz N, Zody M, Anderso N, Biagi T, Patterson N, Pielberg G, Kulbokas E (2003) The dog genome: survey sequencing and comparative analysis. Science 301:1898-1903

Klütsch CF, de Caprona MDC (2010) The IGF1 small dog haplotype is derived from middle eastern grey wolves: a closer look at 
statistics, sampling, and the alleged middle eastern origin of small dogs. BMC Biol 8:119

Larson G, Burger J (2013) A population genetics view of animal domestication. Trends Genet 29:197-205

Larson $\mathrm{G}$ et al (2012) Rethinking dog domestication by integrating genetics, archeology, and biogeography. PNAS 109:8878-8883

Leesch D, Müller W, Nielsen E, Bullinger J (2012) The Magdalenian in Switzerland: re-colonization of a newly accessible landscape. Quat Int 272:191-208

Leonard JA, Wayne RK, Wheeler J, Valadez R, Guillén S, Vila C (2002) Ancient DNA evidence for old world origin of new world dogs. Science 298:1613-1616

Lindblad-Toh K et al (2005) Genome sequence, comparative analysis and haplotype structure of the domestic dog. Nature 438:803-819

Lorenz K (2002) Man meets dog. Psychology Press, Taylor and Francis group, Routeledge, London, New York

Milenkovic M, Sipetic VJ, Blagojevic J, Tatovic S, Vujoševic M (2010) Skull variation in Dinaric-Balkan and Carpathian gray wolf populations revealed by geometric morphometric approaches. J Mammal 91:376-386

Morel P, Müller W (1997) Hauterives-Champréveyres, 11. Un campement magdalénien au bord du lac de Neuchâtel: étude archéozoologique (secteur 1). Archéologie neuchâteloise, 23. Neuchâtel

Morey D, Jaeger R (2015) Paleolithic dogs: why sustained domestication then? J Archaeol Sci: Rep 3:420-428

Napierala H, Uerpmann HP (2012) A 'new' palaeolithic dog from central Europe. Int J Osteoarchaeol 22:127-137

Nehring A (1888) Zur Abstammung der Hunde-Rassen. Zoologische Jahrbücher Abtheilung Systematik, Geographie und Biologie 3:51-58

Oksenberg M (1982) Reconsiderations: a decade of Sino-American relations. Foreign Aff 61:190

Olsen SJ, Olsen JW (1977) The Chinese wolf, ancestor of new world dogs. Science 197:533-535

Ostrander EA, Wayne RK (2005) The canine genome. Genome Res 15:1706-1716

Ovodov ND, Crockford SJ, Kuzmin YV, Higham TF, Hodgins GW, van der Plicht J (2011) A 33,000-year-old incipient dog from the Altai mountains of Siberia: evidence of the earliest domestication disrupted by the last glacial maximum. PLoS One 6:e22821

Pang JF, Kluetsch C, Zo X, Zhang A, Lue L, Angleby H, Ardalan A, Ekström C, Sköllerm A, Lundeber J (2009) mtDNA data indicate a single origin for dogs south of Yangtze river, less than 16,300 years ago, from numerous wolves. Mol Biol Evol 26:2849-2864

Pionnier-Capitan M (2010) La domestication du chien en Eurasie: étude de la diversité passée, approches ostéoarchéologiques, morphométriques et paléogénétiques. $\mathrm{PhD}$ thesis, l'Université de Lyon

Pionnier-Capitan M, Bemilli C, Bodu P, Célérier G, Ferrié J, Fosse P, Garcià M, Vigne JD (2011) New evidence for upper palaeolithic small domestic dogs in South-Western Europe. J Archaeol Sci 38:2123-2140

Pocock R (1946) External and cranial characters of some rare Asiatic mammals recently exhibited by the society. Proc. Zoolog. Soc. Lond 3:310-318

Rütimeyer L (1861) Die Fauna der Pfahlbauten der Schweiz. Geschichte der Wilden und der Haus-Saugetiere. Neue Denkschrift der Algemeinne Schweizerische Geselschaft der ges. Naturwissenschaft, 19, Basel, Switzerland

Sablin M, Khlopachev G (2002) The earliest ice age dogs: evidence from Eliseevichi 11. Curr Anthropol 43:795-799

Savolainen P, Y-p Zhang, Luo J, Lundeberg J, Leitner T (2002) Genetic evidence for an East Asian origin of domestic dogs. Science 298:1610-1613
Savolainen P, Leitner T, Wilton AN, Matisoo-Smith E, Lundeberg J (2004) A detailed picture of the origin of the Australian dingo, obtained from the study of mitochondrial DNA. PNAS 101:12387-12390

Schmitt E, Wallace S (2012) Shape change and variation in the cranial morphology of wild canids (Canis lupus, Canis latrans, Canis rufus) compared to domestic $\operatorname{dog} s$ (Canis familiaris) using geometric morphometrics International. J Osteoarchaeol $24: 42-50$

Schmutz S, Berryere T (2007) Genes affecting coat colour and pattern in domestic dogs: a review. Anim Genet 38:539-549

Schoenebeck JJ, Ostrander EA (2013) The genetics of canine skull shape variation. Genetics 193:317-325

Skoglund P, Götherström A, Jakobsson M (2011) Estimation of population divergence times from non-overlapping genomic sequences: examples from dogs and wolves. Mol Biol Evol 28:1505-1517

Stockhaus K (1965) Metrische Untersuchungen an Schädeln von Wölfen und Hunden. J Zoolog Syst Evol Res 3:157-258

Street M (2002) Ein wiedersehen mit dem hund von Bonn-Oberkassel. Bonner Zoologische Beitrage 50:269-290

Studer T (1901) Die prähistorischen Hunde in ihrer Beziehung zu den gegenwärtig lebenden Rassen. Zurcher und Furrer, Zurich

Sumiński P (1975) Morphologische Unterscheidungsmerkmale zwischen Wolfs-(Canis lupus L.) und Hundeschädel (Canis familiaris L.). Zeitschrift für Jagdwissenschaft 21:227-232

Thalmann O, Shapiro B, Cui P, Schuenemann VJ, Sawyer SK, Greenfield DL, Germonpré MB, Sablin MV, López-Girálde F, Domingo-Roura X (2013) Complete mitochondrial genomes of ancient canids suggest a European origin of domestic dogs. Science 342:871-874

Tsuda K, Kikkawa Y, Yonekawa H, Tanabe Y (1997) Extensive interbreeding occurred among multiple matriarchal ancestors during the domestication of dogs: evidence from inter-and intra-species polymorphisms in the D-loop region of mitochondrial DNA between dogs and wolves. Genes Genet Syst 72:229-238

Vaysse A, Ratnakumar A, Derrien T, Axelsson E, Pielberg G, Sigurdsson S, Fall T, Seppälä E, Hansen M, Lawley C (2011) Identification of genomic regions associated with phenotypic variation between dog breeds using selection mapping. PLoS Genet 7:e1002316

Verginelli F, Capelli C, Coia V, Musiani M, Falchetti M, Ottini L, Palmirotta R, Tagliacozzo A, Mazzorin I, Mariani-Costantin R (2005) Mitochondrial DNA from prehistoric canids highlights relationships between dogs and South-East European wolves. Mol Biol Evol 22:2541-2551

Vila C, Maldonado JE, Wayne RK (1999) Phylogenetic relationships, evolution, and genetic diversity of the domestic dog. J Hered 90:71-77

Vila C, Seddon J, Ellegren H (2005) Genes of domestic mammals augmented by backcrossing with wild ancestors. Trends Genet 21:214-218

Vilà C, Urios V, Castroviejo J (1993) Tooth losses and anomalies in the wolf (Canis lupus). Can J Zool 71:968-971

Vilà C, Savolainen P, Maldonado J, Amorim I, Rice J, Honeycutt R, Crandall K, Lundeberg J, Wayne R (1997) Multiple and ancient origins of the domestic dog. Science 276: 1687-1689

Vonholdt B, Pollinger J, Lohmueller K, Han E, Parker H, Quignon P, Degenhardt J, Boyko A, Earl D, Auton A, Reynolds A (2010) Genome-wide SNP and haplotype analyses reveal a rich history underlying dog domestication. Nature 464:898-902

Wayne RK (2012) Evolutionary genomics of dog domestication. Mamm Genome 23:3-18 
Wayne RK, Ostrander EA (1999) Origin, genetic diversity, and genome structure of the domestic dog. BioEssays 21:247-257

Wayne RK, Ostrander EA (2007) Lessons learned from the dog genome. Trends Genet 23:557-567
Wolfgram A (1894) Die Einwerkung der Gefangenschaft auf die Gestaltung des Wolfschädels. Zoologisches Jahrbuch (Abteilung fur Systematik) 7:773-822 